\title{
Computer Simulation of Fast Neutron Multiplicity Analysis
}

\author{
Lin Zhuang ${ }^{1,}$, , Quanhu Zhang ${ }^{1}$, Wenming Zuo ${ }^{1}$, Chen Chen ${ }^{1}$ \\ ${ }^{1} X i$ 'an institute of High Tech Research, Xi'an 710025, China \\ a1838953291@qq.com
}

KEYWORD: Fast neutron, Multiplicity, GEANT4

ABSTRACT: The paper constructs the model of nuclear materials and fast neutron multiplicity counter, and obtains the mass of nuclear materials by utilizing the equation of fast neutron multiplicity. Via GEANT4 tool kit, the paper obtains the simulated results which are identical well to the results calculated by the equation. This indicates the validity and feasibility of computer simulation of fast neutron multiplicity analytical method and lays the foundation of the design and application of fast neutron multiplicity counter.

\section{Introduction}

The certification of the properties of the closed container core material has been an important issue in the field of nuclear safeguards and nuclear disarmament verification. Neutron Multivariate Measurement and Analysis ${ }^{[1]}$ is a very important non-destructive analysis technique, which is mainly used to analyze the quality properties of sealed containers and materials. The detector used in this technique is generally a ${ }^{3} \mathrm{He}$ detector, but due to the current high cost of ${ }^{3} \mathrm{He}^{[2-3]}$, and the detection of moderated neutrons, the time information and energy information loss is serious, and thus a number of alternative technologies 。

Based on liquid flash fast neutron multiple analysis technology is one of the alternative technology, the current development of the technology is not yet mature. Although the fast neutron multiplicity analysis technique based on liquid scintillation is relatively low in detection efficiency, it detects fast neutrons that are not moderated and retains the time information and energy information of the measured neutrons well, and the cost Is relatively cheap, so it can be a good alternative to ${ }^{3} \mathrm{He}$ detector based neutron multiple measurement technology. The study of fast neutron meta-measurements was first seen in the 1980s, and the Los Alamos National Laboratory (LANL) in the United States constructed a fast neutron counting prototyping device for the analysis of non-pure plutonium materials, consisting of four liquid flickers Body detector, the structure shown in Fig.1. The upper layer is the sample measurement chamber and the liquid scintillation detector, the middle is the pulse shape discrimination logic circuit, the bottom is the signal and the computer processing system..

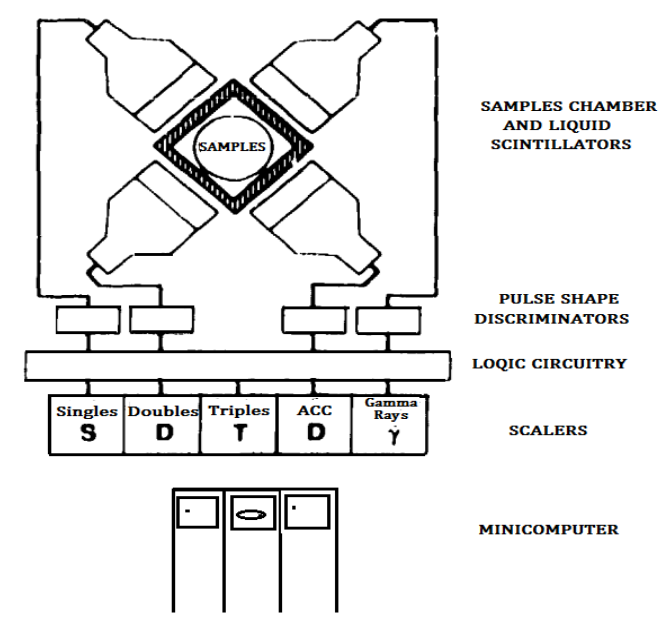

Fig.1 LLNL The earliest fast neutron met count measurement prototype device 
This paper mainly uses GEANT4 simulation software to simulate the fast neutron multiplicative technology based on liquid scintillation. Using simulation results can be used to verify the correctness of the measurement and analysis equations. Simulation measurements can save hardware costs and use GEANT4 for analog measurements to record the physical quantities during the test and facilitate correlation analysis.

\section{Quantum Description of Fast Neutron Multiplicity}

The neutron multiplicity analysis method can be divided into thermal neutron multiple analysis technique and fast neutron multiple gravity analysis technique according to its detector. The neutron multiplicity analysis method is based on the fact that plutonium material emits 0 to 8 unequal neutrons at fission.

The neutron detector uses the foreground counter and the background counter for counting, and the multiplicity of the foreground counter contains both the contribution of the neutron associated with the trigger neutron and the neutron contribution independent of the trigger neutron, and the background counter $(S, D, T)$ can be used to calculate the neutron multiplicity distribution associated with the trigger neutrons using the count distribution of the two sets of counters. The three most direct factors affecting the neutron emission of plutonium are $F, M$ and $\alpha$, ie the spontaneous fission rate, self-propagating factor and $(\alpha, n)$ reaction coefficient of the sample. In order to obtain the quality, must be required for F, M and $\alpha$ three parameters. According to the basic principle of the matrix equation, the three unknowns are required to obtain at least three or more solving equations. Neutron multiplicity analysis method is by measuring $S, D, T$ is listed three equations ${ }^{[4]}$, so as to solve $\mathrm{F}, \mathrm{M}$ and $\alpha$. According to the mathematical function of probability mother function and factorial moment, $\mathrm{S}$, $\mathrm{D}$ and $\mathrm{T}$ satisfy the following equations.

$$
\begin{aligned}
S= & F \varepsilon(1+\kappa) v_{s f 1}(1+\alpha) M \\
D= & \frac{F \varepsilon^{2}(1+\kappa)^{2} f_{d} M^{2}}{2}\left[v_{s f, 2}+\left(\frac{M-1}{v_{i 1}-1}\right) v_{s f, 1}(1+\alpha) v_{i 2}\right]+F \varepsilon \kappa f_{d} v_{s f, 1}(1+\alpha) M \\
T= & \frac{F \varepsilon^{3}(1+\kappa)^{3} f_{t} M^{3}}{6}\left[v_{s f, 3}+\left(\frac{M-1}{v_{i 1}-1}\right) 3 v_{s f, 2} v_{i 2}+\left(\frac{M-1}{v_{i 1}-1}\right) v_{s f, 1}(1+\alpha) v_{i 3}\right. \\
& \left.+3\left(\frac{M-1}{v_{i 1}-1}\right)^{2} v_{s f, 1}(1+\alpha) v_{i 2}^{2}\right]+F \varepsilon^{2} \kappa(1+\kappa) f_{t} M^{2}\left[v_{s f, 2}+\left(\frac{M-1}{v_{i 1}-1}\right) v_{s f, 1}(1+\alpha) v_{i 2}\right]
\end{aligned}
$$

Where:

$$
\begin{aligned}
& v_{s f, 1}-{ }^{240} \mathrm{PuFirst} \text { order factor of spontaneous fission; } \\
& v_{s f, 2}-{ }^{240} \mathrm{Pu} \text { Second order factorial moments of spontaneous fission; } \\
& v_{s f, 3}-{ }^{240} \mathrm{Pu} \text { Third order factorial moments of spontaneous fission; } \\
& v_{i 1}-{ }^{239} \mathrm{Pu} \text { The First Order Factor of Induced Fission; } \\
& v_{i 2}-{ }^{239} \mathrm{Pu} \text { the second order factor of the fission multiplicity; } \\
& v_{i 3}-{ }^{239} \mathrm{Pu} \text { triggering the third order factor of fission multiplicity; } \\
& f_{d} \text { - double counting rate (Doubles) gate width factor; } \\
& f_{t} \text {-triple count factor (Triples) of the door width factor; } \\
& \kappa \text { - scattering crosstalk rate; } \\
& \varepsilon \text { - detection efficiency. } \\
& \text { By substituting } F \text { and } \alpha \text { for the mutual substitutions of equations }(1),(2),(3), \text { a commutative cubic }
\end{aligned}
$$$$
\text { equation about } \mathrm{M} \text { is obtained, as shown in (4), where } \mathrm{a}, \mathrm{b}, \mathrm{c} \text { are known by the known parameters, } \mathrm{M}
$$$$
\text { solution can be obtained by solving the one yuan cubic equation. }
$$$$
a+b M+c M^{2}+M^{3}=0
$$ 
After solving M, the values of $F$ and $\alpha$ are solved by the equation（5） and (6)

$$
\begin{aligned}
F & =\frac{\frac{2}{f_{d}}\left[\frac{D}{\varepsilon(1+\kappa)}-\frac{\kappa}{\varepsilon(1+\kappa)^{2}} S\right]-\frac{M(M-1) v_{i 2} S}{v_{i 1}-1}}{\varepsilon(1+\kappa) M^{2} v_{s f, 2}} \\
\alpha & =\frac{S}{F \varepsilon(1+\kappa) v_{s f, 1} M}-1
\end{aligned}
$$

The equivalent ${ }^{240} \mathrm{Pu}$ mass of the sample can then be determined from the value of the spontaneous fission rate F obtained and the number of fractions per second of ${ }^{240} \mathrm{Pu}$.

\section{Simulation study}

Simulation with GEANT4 can verify the correctness of the FNMC equation by simulation. The simulation can save the cost of the hardware. The parameters of the sample can be set arbitrarily during the measurement and some of the intermediate quantities can be recorded. The simulation flow chart is shown in Fig.2.

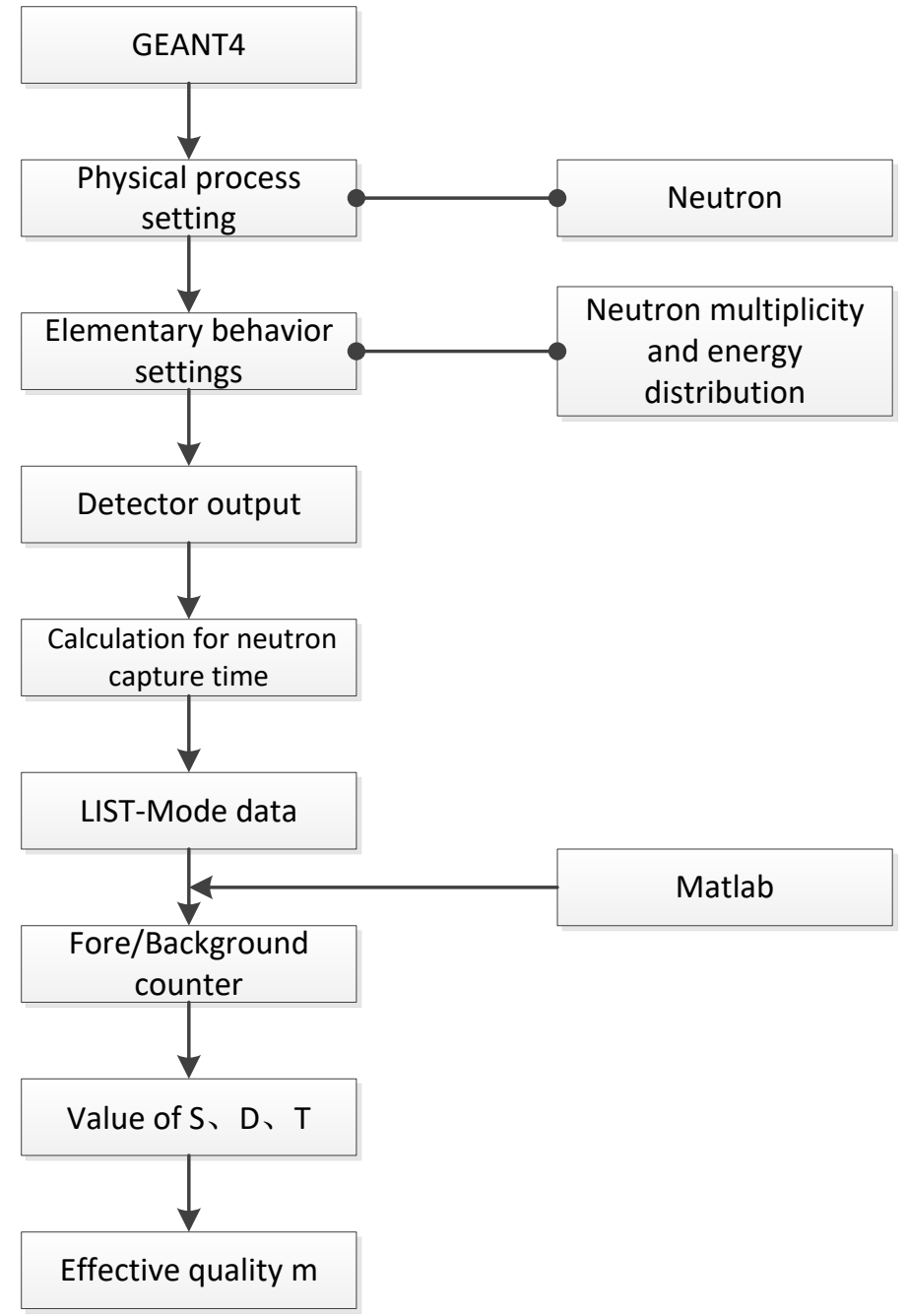

Fig. 2 simulation flow chart

Scattering crosstalk simulation scale

The thermal neutron detector detects neutrons by radiation trapping, so there is no crosstalk problem. However, the principle of fast neutron detection using organic liquid scintillation detectors is to detect neutron scattering of the recoil protons. During neutron scattering, neutrons do not dissipate all of the energy, and the scattered neutrons are still likely to fly to another detector and respond, resulting in a 
high count error. For subsequent accurate calculations, it is necessary to quantitatively calculate the scattered crosstalk.

Since scatter crosstalk is a parameter that can not be obtained experimentally, researchers have learned that scatter crosstalk is present ${ }^{[5]}$ and has some effect on the measurement results. However, due to the lack of an effective method for estimating the effect of scattering crosstalk, Generally only in the experimental instrument layout to optimize, in order to minimize the probability of scattering crosstalk. In the measurement process, the higher the detection efficiency, the higher the scattering crosstalk rate. In order to maintain good detection efficiency, only through quantitative simulation to get accurate results.

The model of the measurement device used in the simulation study (the scintillator position shown in Fig.3) was established by reference to the model in reference [6]. The geometric layout of the measuring device is a $3 \times 8$, uniformly wound $\emptyset 5$ "x $2 "$ " BC-501-A liquid scintillator detector with a detector surface distance of $20 \mathrm{~cm}$ from the center axis.

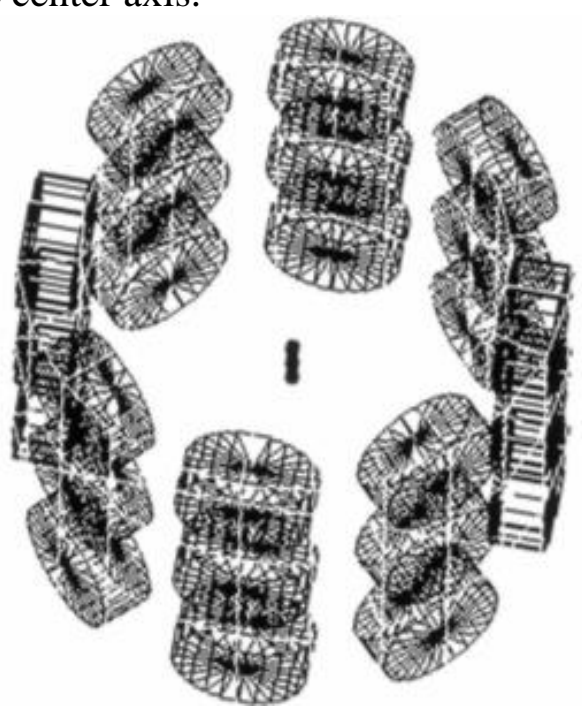

Fig. 3 Measuring device model

In the study of the scattered crosstalk simulation, the actual behavior of each event is not set. The primary behavior of each event is set as follows: every time the ${ }^{240} \mathrm{Pu}$ spontaneous fission spectrum is emitted, a neutron is emitted randomly in the random position of the sample region, and each event is detected. Of the neutron signal multiplicity, the simulation results shown in Table 1.

Table 1 Distribution of Neutron Signals Caused by Single Neutron

\begin{tabular}{c|c|c|c|c|c}
\hline $\begin{array}{c}\text { Signal } \\
\text { multiplici- } \\
\text { ty }\end{array}$ & 0 & 1 & 2 & 3 & Total \\
\hline count & $9,014,214$ & 980,210 & 5,560 & 16 & $10,000,000$ \\
\hline
\end{tabular}

Ideally, if there is no scattered crosstalk, a neutron can only produce a neutron signal at most. However, it can be seen from Table 1 that using a fast neutron multiplicity measuring device, a neutron may cause the detector to output two or even three neutron signals, which is mainly the cause of the scattered crosstalk. Assuming that each neutron has a signal in the first probe, the probability of $\kappa$ is generated in the second probe ( $\kappa$ can be called the scattered crosstalk rate).

According to the simulation results, the problem is simplified by ignoring a neutron-induced response of more than two detectors. According to the simulation results, for the measurement device model of Fig. 3, the scattering crosstalk rate $\kappa$ is $(5,560+16) /(980,210+5,560+16)=5.66 \%$.

Metal plutonium simulation

Simulation settings

Use the GEANT4 toolbox for simulation of the sample. In the measurement, the plutonium material is placed in the center of the counter. The primary firing of each event is the spontaneous fission neutron 
and $(\alpha, n)$ reactive neutrons. The time at which the primary triggering occurs is randomly distributed according to the actual time distribution rule. The time at which the signal is detected is recorded in list mode, and then the signals are processed using the Matlab software.

A simulated measurement of $82.9 \mathrm{~g}$ of metallic plutonium $\left(90 \%{ }^{240} \mathrm{Pu}, 10 \%{ }^{239} \mathrm{Pu}, 19.8 \mathrm{~g} / \mathrm{cm}^{3}\right)$. Simulated measurements simulated $4,000,000$ primary behaviors (all ${ }^{240} \mathrm{Pu}$ spontaneous fission), and the output data contained 922,160 detections of neutrons. According to the time to sort the data, in order to ensure the accuracy of the data, access to $90 \%$ of the middle of the data (from 46,101 to $875,542)$, the corresponding measurement time length of $101.9 \mathrm{~s}$.

Data acquisition and processing

At the time of detection, in order to fully reflect the time correlation between the detected neutrons, to eliminate the interaction between the neutrons generated by different source events, the foreground counter and the background counter are used to count the way. The multiplicity in the foreground counter contains both the contribution of the neutron associated with the trigger neutron and the contribution of the neutrons independent of the trigger neutron, and the multiplicity in the background counter is all neutrons that are independent of the trigger neutron, The neutron multiplicity distribution associated with the trigger neutron can be deduced using the count distribution of the two sets of counters. By programming the foreground counter and the background counter, the count of the neutron count multiplicity is shown in Table 2.

Table 2 Metal plutonium simulation measurements

\begin{tabular}{c|c|c|c|c|c|c|c|c}
\hline $\begin{array}{c}\text { Count } \\
\text { Multiplicity }\end{array}$ & 0 & 1 & 2 & 3 & 4 & 5 & 6 & 7 \\
\hline $\begin{array}{c}\text { Foreground } \\
\text { count } \\
\text { distribution }\end{array}$ & 0.8864 & 0.1015 & 0.0106 & 0.0013 & $1.88 \mathrm{E}-4$ & $2.41 \mathrm{E}-5$ & $6.03 \mathrm{E}-6$ & $1.21 \mathrm{E}-6$ \\
\hline $\begin{array}{c}\text { Background } \\
\text { count } \\
\text { distribution }\end{array}$ & 0.9993 & $6.60 \mathrm{E}-4$ & $7.29 \mathrm{E}-5$ & $7.30 \mathrm{E}-6$ & $1.20 \mathrm{E}-6$ & 0 & 0 & 0 \\
\hline
\end{tabular}

The values of $\mathrm{S}, \mathrm{D}$, and $\mathrm{T}$ are obtained according to the order factor.

$$
\begin{aligned}
& S=\frac{875,522-46,108}{101.9}=8.14 \times 10^{3} \\
& D=S\left(f_{1}-b_{1}\right)=1.03 \times 10^{3} \\
& T=\frac{1}{2} S\left[\left(f_{2}-b_{2}\right)-2 b_{1}\left(f_{1}-b_{1}\right)\right]=1.28 \times 10^{2}
\end{aligned}
$$

Since the detection efficiency $(\varepsilon)$ of the organic scintillator detector varies with the incident neutron energy and the neutron energy is attenuated during the transport of the sample, the $\varepsilon$ used in the calculation is not a pre-scaled value, But by the simulation data. The ratio of the total signal number to the total neutron number in the simulation (including the source neutron and the induced fission neutron) is divided by $(1+\kappa)$. The self-propagating coefficient $(\mathrm{M})$ of the sample is also given by the simulation data, $\mathrm{M}$ is 1.149. Calculate according to the theoretical calculation step (since the sample is metal plutonium, so $\alpha=0$ ).

$$
F=\frac{\frac{2}{f_{d}}\left[\frac{D}{\varepsilon(1+\kappa)}-\frac{\kappa}{\varepsilon(1+\kappa)^{2}} S\right]-\frac{M(M-1) v_{i 2} S}{v_{i 1}-1}}{\varepsilon(1+\kappa) M^{2} v_{s f, 2}}=3.24 \times 10^{4}
$$

Plutonium effective quality:

$$
m_{240}=\frac{F}{(473 \text { fission } / s \cdot g)}=68.50
$$

Because the sample is $82.9 \mathrm{~g}$ metal plutonium $\left(90 \%{ }^{240} \mathrm{Pu}, 10 \%{ }^{239} \mathrm{Pu}\right)$, considering the ${ }^{240} \mathrm{Pu}$ isotopic composition of $90 \%$, and finally can be found in plutonium quality relative deviation of $8.1 \%$, the solution is better. 


\section{Conclusion}

In this paper, 24 models of liquid level detector are constructed by referring to the more advanced fast neutron multiplicity analysis experiment device, and the whole process simulation of metal plutonium is carried out. The simulation results are in good agreement with the reference value of the sample, Which shows the correctness and feasibility of computer simulation of fast neutron multiplicity analysis method, which lays the foundation for the design and application of fast neutron multiplicity counter.

\section{Literature References}

[1] Ensslin N, Harker WC, Krick, MS, et al. Application guide to neutron multiplicity counting [J], Los Alamos Report La-13422-M,1998.

[2] Bentz J, Founds N, Glaser J, et al. US Government Approach to Addressing the He-3 Shortage [J]. Procceedings of the Institute of Nuclear Materials Management Ann, 2010.

[3] Shea DA, Morgan D. The Helium-3 Shortage: Supply, Demand, and Options for Congress [C], 2010.

[4] Equvist A, Safeguards: Modelling of the Detection and Characterization of Nuclear Materials [D]. Goteborg: Chalmers University Of Technology, 2010.

[5] Sufen Li, Suizheng Qiu, Quanhu Zhang, et al. Fast-neutron multiplicity analysis on liquid scintillation [J]. Applied Radiation and Isotopes, 2016

[6] Chichester D, Pozzi S, Dolan J, et al. MPACT Fast Neutron Multiplicity System Design Concepts [J]. Idaho National Laboratory, Idaho Falls, ID, 2012. 